\title{
Análisis computacional de un ensayo de flexión por 3 puntos de un material sándwich natural compuesto de fibra de ichu y núcleo de corcho aglomerado
}

\author{
David O. Sagástegui Calderón \\ Universidad Nacional de Ingeniería, Av. Túpac Amaru 210, Rímac 15333, Lima, Perú \\ Enviado el 14 de noviembre del 2021. Aceptado el 26 de diciembre del 2021.
}

DOI: https://doi.org/10.33017/RevECIPeru2021.0010/

\section{Resumen}

Hoy en día las tecnologías sostenibles con el medio ambiente están obteniendo una gran implicancia en las diversas industrias. En el presente artículo, se propone a los materiales compuestos naturales como alternativa al uso de los materiales compuestos sintéticos empleados en aplicaciones aeroespaciales, estructurales, construcciones navales, automovilísticas, etc [1]. Esta alternativa es tema de interés en los investigadores como se puede comprobar en las recientes investigaciones sobre materiales compuestos naturales como la fibra de lino [2] y la fibra de ichu [3],[4], que presentan propiedades mecánicas comparables e incluso superiores a la fibra de vidrio. Basado en aquellas investigaciones, se plantea el objetivo de este presente artículo de proponer un material sándwich compuesto de laminados de fibra de ichu y un núcleo de corcho, combinando en este sentido sus propiedades, con la finalidad de obtener un compuesto natural altamente resistente a los esfuerzos de flexión. En los resultados se expondrá el campo de esfuerzos según el criterio de Von Mises, el campo de deformaciones y el campo de esfuerzos máximos, donde se podrá observar que, según el análisis computacional desarrollado, el material sándwich presenta un comportamiento estructural dependiente de la orientación de las fibras en las capas del laminado.

Descriptores: corcho, ichu, flexión, material compuesto, natural, sándwich, sostenibles.

\begin{abstract}
Today, environmentally sustainable technologies are obtaining great implications in various industries. In this paper, natural composite materials are proposed as an alternative to the use of synthetic composite materials used in aerospace, structural, shipbuilding, automotive applications, etc. [1]. This alternative is a subject of interest to researchers, as can be seen in recent research about natural composite materials such as A. [2] and ichu fiber [3],[4], which have mechanical properties that are comparable and even superior to those of fiberglass. Based on those investigations, the objective of this present paper is to propose a sandwich material composed of ichu fiber laminates and a cork core, combining in this sense their properties, in order to obtain a highly resistant natural compound to the bending stresses. The results will show the stress field according to the Von Mises criterion, the deformation field and the máximum stress field, where it can be observed that, according to the computational analysis developed, the sandwich material presents a structural behavior dependent on the orientation of the fibers in laminated layers.
\end{abstract}

Keywords: bending, composite material, cork, ichu fiber, natural, sandwich, sustainable. 


\section{Introducción}

El planeta Tierra, afronta un gran problema contra el exceso de plástico, es por ello que en la búsqueda de hacerle frente a esa problemática se ha empezado a investigar materiales sustitutos. En este presente artículo se propone como alternativa el uso en conjunto de laminados de fibra de ichu y núcleo de corcho, conformando en este sentido un material con una estructura tipo sándwich. Estos materiales naturales, en la actualidad vienen siendo temas de interés por los investigadores debido a sus propiedades mecánicas y térmicas que se han podido obtener, como expone la investigadora Sandra Mori y sus colegas, en sus artículos de investigación [3],[4], donde se evidencia que la fibra de ichu mostró características sobresalientes como alta resistencia, alto módulo y un excelente comportamiento como refuerzo de polímeros [3]. Complementariamente, el Ing. Cristian Córdova plantea el uso de laminados de fibra de ichu como una prometedora alternativa para la elaboración de prótesis transtibiales [5], ya que, de dicha investigación concluye que las prótesis elaboradas con laminado de ichu, cumplen con la funcionalidad estructural deseada. En la industria naval, el PhD. José Luis Mantari, ha propuesto a la fibra de ichu, como material de construcción en los cascos de las embarcaciones [6].

\section{Metodología}

Para el análisis computacional se ha empleado el método de elementos finitos en el software ABAQUS. Además, se ha empleado como referencia para el ensayo de flexión la norma ASTM E290, que es la norma que rige en este tipo de ensayos [9]. Complementariamente, en esta sección, se detalla las propiedades de los materiales empleados en el modelamiento numérico, así como las condiciones de contorno a las que está sometida la simulación, siendo estas muy importantes, ya que, tienen influencia directa en los resultados de la simulación.

\subsection{Materiales}

Para el material sándwich, como se muestra en la Figura 1, se ha estructurado capas de laminado de fibra y un núcleo. Este tipo de materiales ofrece mayores ventajas en sus propiedades, en
El investigador Osvaldo Castro, propone el corcho aglomerado como alternativa de núcleo ideal en un material sándwich [7], basado en los resultados de sus investigaciones las cuales arrojan que después de haber efectuado un ensayo de flexión por 3 puntos, un ensayo de impacto y un ensayo de conductividad térmica, el corcho aglomerado presenta excelentes propiedades como un buen esfuerzo cortante, una excelente capacidad de recuperación ante los impactos y una gran capacidad para comportarse como aislante térmico. En este presente artículo de investigación, se ha desarrollado un análisis computacional de un ensayo de flexión por 3 puntos, empleando el método de elementos finitos, el cual permitirá predecir el comportamiento estructural del material sándwich en estudio, al ser sometido a una carga con desplazamiento prescrito ocasionando que el material se flexione y demuestre computacionalmente su capacidad de soportar la carga a la que está sometido.

Finalmente se presentan los resultados del campo de esfuerzos soportados por el material, el cuál arroja un valor máximo de $230 \mathrm{MPa}$, lo que indica que el material sándwich al combinar sus propiedades mecánicas da como resultado un material resistente en comparación con los compuestos laminados reforzados con fibra de vidrio [8], al estar sometido a esfuerzos de flexión.

comparación a los materiales compuestos conformado únicamente por capas de laminado, como se observa en la Figura 2.

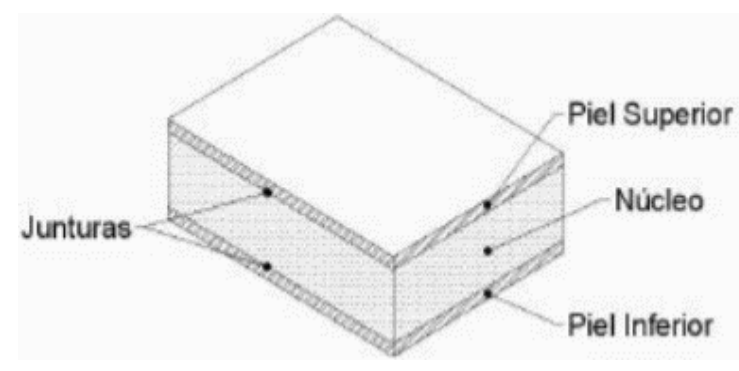

Figura 1: Estructura característica de un material sándwich [10] 


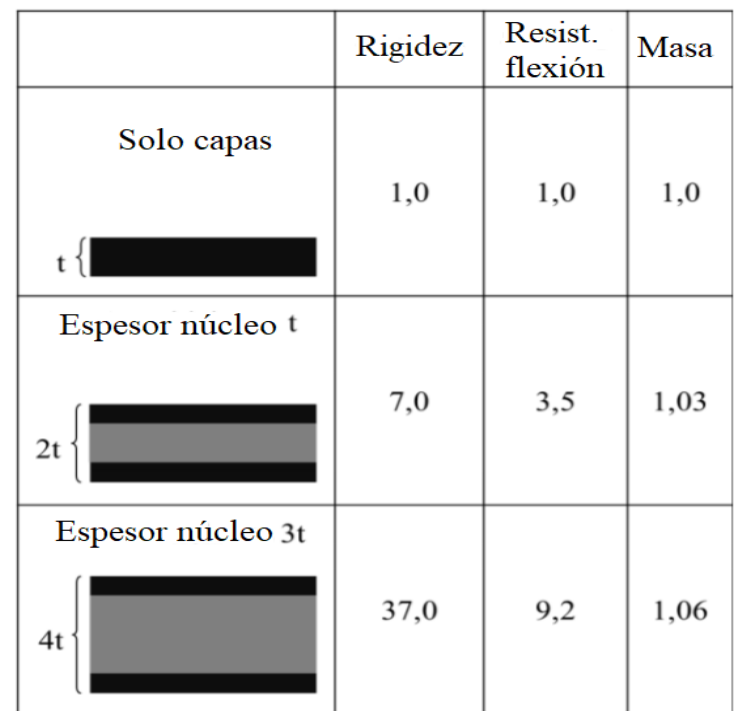

Figura 2: Comparación de la rigidez, resistencia a la flexión y masa, entre las diversas configuraciones de material sándwich [11]

Las pieles están hechas de fibra de ichu natural y resina de poliéster, que juntos conforman un laminado, con atractivas propiedades mecánicas, como puede verse en la Tabla 2 [5]. En el caso de la fibra de ichu, que es el material innovador que se está introduciendo en la presente investigación, es compuesto por varios componentes químicos, que se pueden ver en la Tabla 1 [3], siendo la celulosa la más importante en las fibras naturales, ya que influye en las propiedades mecánicas y se utiliza como aislamiento térmico y acústico en otras aplicaciones. Además, la fibra Ichu tiene un comportamiento ortotrópico [3].

Tabla 1: Composición química de la fibra Stipa Ichu [3]

\begin{tabular}{lcc}
\hline Componentes & $\begin{array}{c}\text { Porcentaje } \\
(\%)\end{array}$ & $\begin{array}{c}\text { Desviación } \\
\text { Estándar }(\%)\end{array}$ \\
\hline Celulosa & 38.07 & 0.91 \\
\hline Hemicelulosa & 26.52 & 1.21 \\
\hline Lignina & 15.56 & 0.72 \\
\hline $\begin{array}{l}\text { Otros } \\
\text { extractivos }\end{array}$ & 14.57 & 0.06 \\
Cenizas & 4.36 & 0.17 \\
\hline Total & 99.08 & - \\
\hline Humedad & 9.33 & 0.12 \\
\hline
\end{tabular}

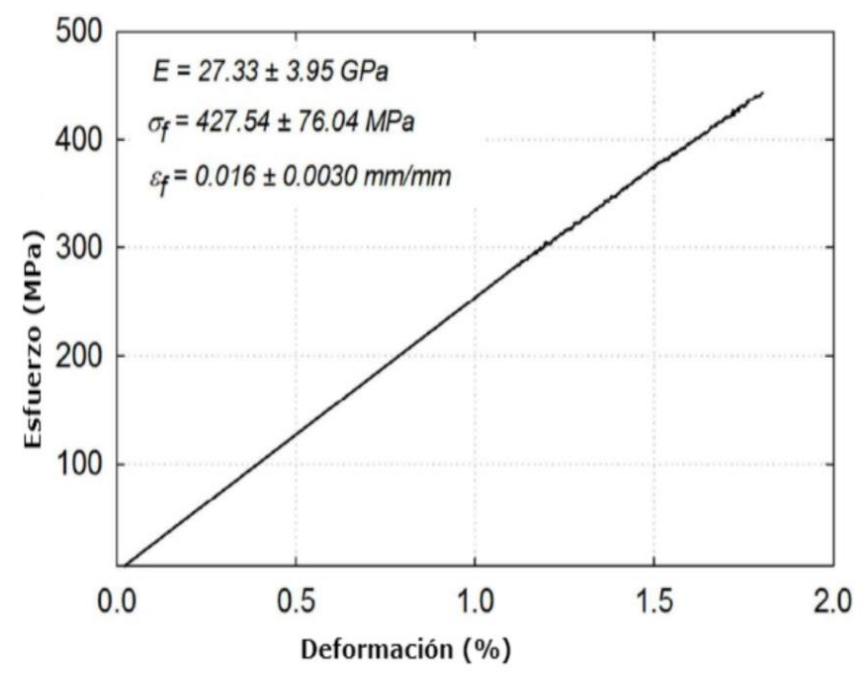

Figura 3: Curva típica de esfuerzo-deformación de la fibra Stipa Ichu [5]

Tabla 2: Propiedades mecánicas de la matriz y de la fibra Stipa Ichu [5]

\begin{tabular}{ccc}
\hline Parámetros & Resina Poliéster & Stipa Ichu \\
\hline E11(GPa) & 2.8 & 27.3 \\
\hline E22(GPa) & 2.8 & 7.88 \\
\hline E33(GPa) & 2.8 & 8.72 \\
\hline $\boldsymbol{v}_{\mathbf{1 2}}$ & 0.3773 & 0.1897 \\
\hline $\boldsymbol{v}_{\mathbf{2 3}}$ & 0.3773 & 0.22 \\
\hline $\boldsymbol{v}_{\mathbf{1 3}}$ & 0.3773 & 0.389 \\
\hline $\boldsymbol{\rho}\left(\mathbf{k g} / \mathbf{m}^{\mathbf{3}}\right)$ & 1270 & 1447 \\
\hline
\end{tabular}

Tabla 3: Propiedades mecánicas del compuesto laminado de fibra de ichu [5]

\begin{tabular}{lc}
\hline Parámetros & Valores \\
\hline Módulo de flexión longitudinal (GPa) & 8 \\
\hline $\begin{array}{l}\text { Módulo de flexión transversal (GPa) } \\
\text { Esfuerzo de flexión longitudinal }\end{array}$ & 2.88 \\
$\begin{array}{l}(\mathrm{MPa}) \\
\begin{array}{l}\text { Esfuerzo de flexión transversal } \\
(\mathrm{MPa})\end{array}\end{array}$ & 33 \\
\hline Densidad $\left(\mathrm{kg} / \mathbf{m}^{\mathbf{3}}\right)$ & 1340 \\
\hline
\end{tabular}

Con la finalidad de obtener resultados con mayor precisión, se tomarán los valores del laminado de fibra de ichu expuestos en la Tabla 4, ya que, detalla los valores de sus propiedades mecánicas, teniendo en cuenta el comportamiento ortotrópico de la fibra. 
Tabla 4: Propiedades ortotrópicas del laminado [5]

\begin{tabular}{cc}
\hline Parámetros & Valor \\
\hline E11(GPa) & 10.558 \\
\hline E22(GPa) & 4.051 \\
\hline E33(GPa) & 4.158 \\
\hline $\boldsymbol{v}_{\mathbf{1 2}}$ & 0.3322 \\
\hline $\boldsymbol{v}_{\mathbf{2 3}}$ & 0.3328 \\
\hline $\boldsymbol{v}_{\mathbf{1 3}}$ & 0.4671 \\
\hline E12(GPa) & 1.342 \\
\hline E23(GPa) & 1.259 \\
\hline E13(GPa) & 1.485 \\
\hline
\end{tabular}

Para la elección del material del núcleo, se debe tener en cuenta que esta parte del material sándwich debe ser un material ligero, razón por la cual se ha seleccionado el corcho aglomerado como material ideal para el núcleo del material sándwich del presente estudio. Además, posee excelentes propiedades mecánicas y térmicas, especialmente su módulo de cizallamiento, baja absorción de líquidos y baja densidad. Sus propiedades mecánicas y térmicas se exponen en la Tabla 5.

Tabla 5: Propiedades mecánicas y térmicas del corcho aglomerado [12]

\begin{tabular}{lc}
\hline Parámetros & Valor \\
\hline Densidad $\left(\mathrm{kg} / \mathbf{m}^{3}\right)$ & 140 \\
\hline Esfuerzo de compresión (MPa) & 0.3 \\
\hline Módulo de compresión (MPa) & 5.1 \\
\hline Fuerza de Tensión (MPa) & 0.6 \\
\hline Esfuerzo de corte $(\mathrm{MPa})$ & 0.9 \\
\hline Módulo de corte $(\mathrm{MPa})$ & 5.9 \\
\hline Conductividad térmica $(\mathrm{W} / \mathrm{mK})$ & 0.042 \\
\hline Factor de pérdida $(\mathbf{a} \mathbf{1 k H z})\left(\mathbf{N} / \mathbf{c m}^{3}\right)$ & 0.022 \\
\hline
\end{tabular}

\subsection{Modelamiento Numérico}

Para el modelamiento numérico del ensayo de flexión, se emplea el esquema recomendado por la norma ASTM E290 (Figura 4). Establecido el modelo, se procede a modelar el esquema en el software de elementos finitos. El esquema estará conformado por un material sándwich con 4 capas de laminado de $300 \mathrm{~mm} \times 80 \mathrm{~mm} \times 2 \mathrm{~mm}$ tanto como en la parte superior e inferior del material, el núcleo de corcho contará con las medidas de $300 \mathrm{~mm} \times 80$ $\mathrm{mm} \times 20 \mathrm{~mm}$ y los apoyos junto con la carga cuentan con un diámetro de $15 \mathrm{~mm}$ y $80 \mathrm{~mm}$ de profundidad. Posteriormente se establece las condiciones de contorno (Tabla 6) a las que estará sometido el esquema. Dentro de las condiciones de contorno se establecen dos condiciones "tie" que representa una unión o contacto entre las pieles del sándwich y el núcleo, y además 3 puntos remotos en el centro de los apoyos y de la carga. Estos puntos remotos son una forma de conexión con el modelo que facilitan la definición de las condiciones de contorno en la estructura. Finalmente se realiza el mallado, y se efectúa la simulación.

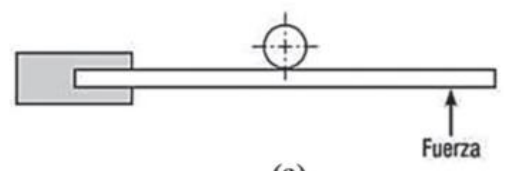

(a)

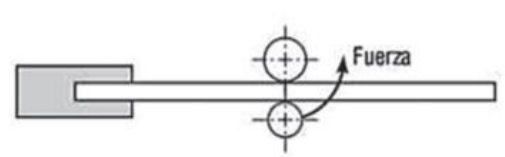

(b)

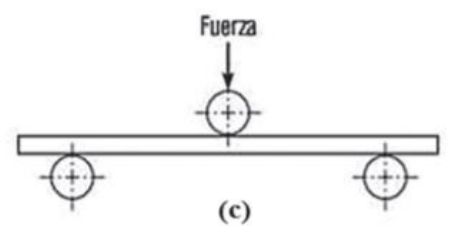

(c)

Figura 4: Esquemas para pruebas de flexión según la norma ASTM [9]

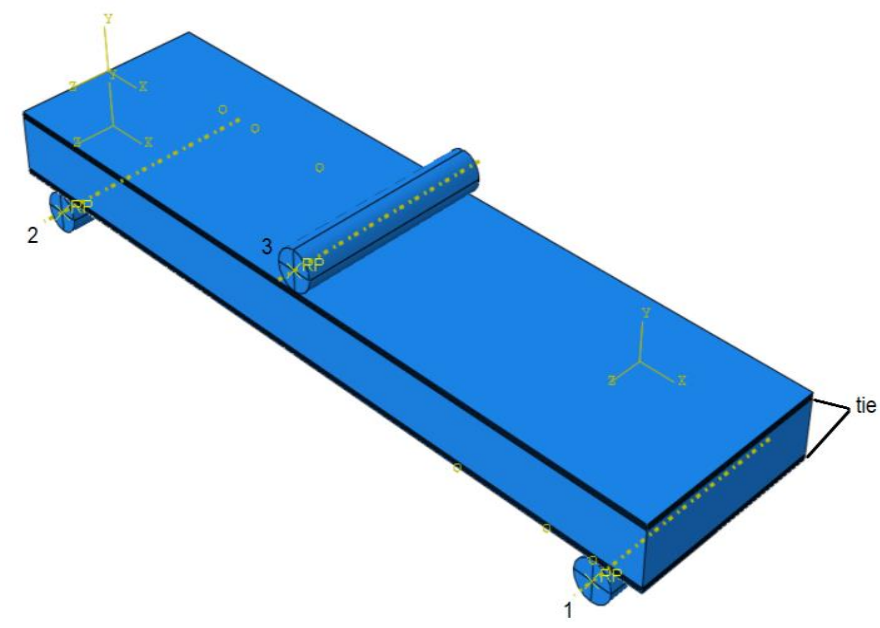

Figura 5: Modelado geométrico del ensayo de flexión en el software Abaqus (Fuente: propia)

Tabla 6: Condiciones de contorno de la estructura (Fuente: propia)

\begin{tabular}{lc}
\hline Posición & Condición \\
\hline $\mathbf{1}$ & Fija en los 3 ejes \\
\hline $\mathbf{2}$ & Fija en los 3 ejes \\
\hline $\mathbf{3}$ & Fija en los ejes $x, z$ y en el eje y se \\
& desplaza $50 \mathrm{~mm}$ \\
\hline
\end{tabular}


Debido al comportamiento ortotrópico de la fibra de ichu, se ha otorgado una orientación distinta según cada laminado. Para la piel superior la orientación de las fibras para los 4 laminados es [0, -45,45,90] y para la piel secundaria será $[90,45,-45,0]$.

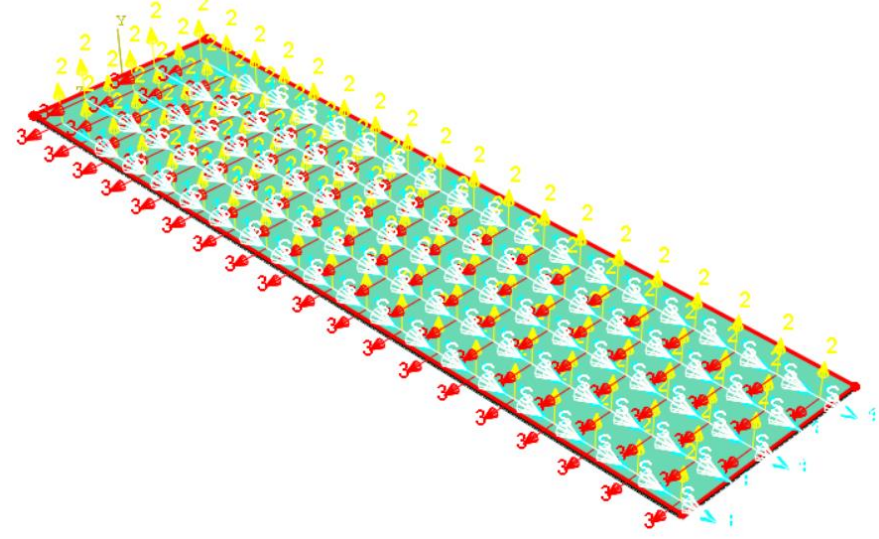

Figura 6: Orientación a $0^{\circ}$ de las fibras en la capa de laminado (Fuente:propia)

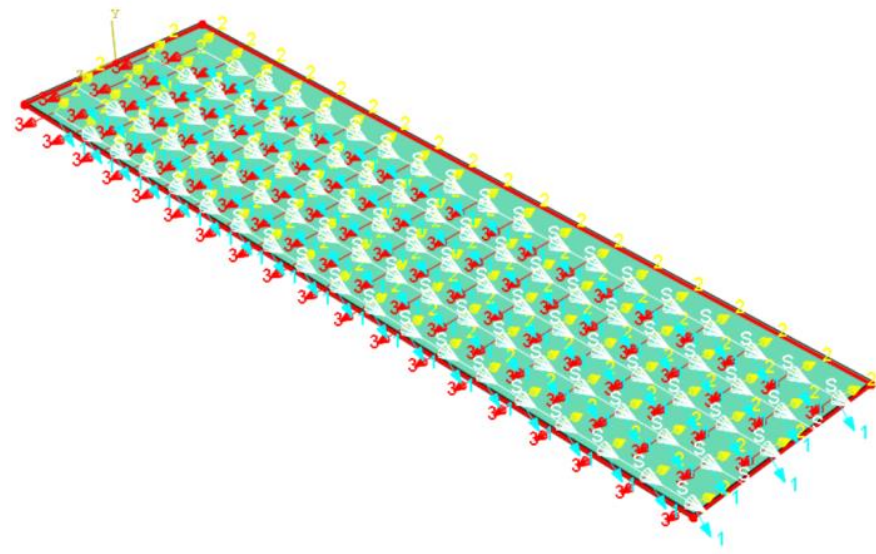

Figura 7: Orientación a $-45^{\circ}$ de las fibras en la capa de laminado (Fuente: propia)

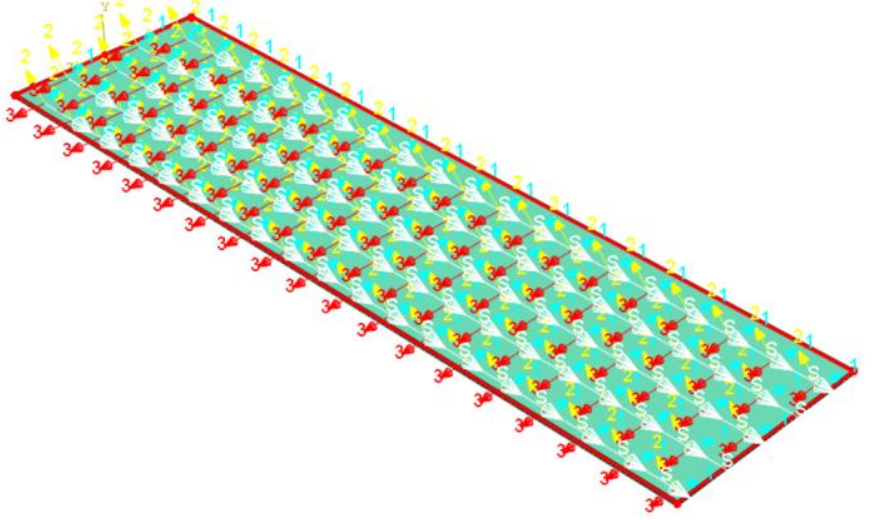

Figura 8: Orientación a $45^{\circ}$ de las fibras en la capa de laminado (Fuente: propia)

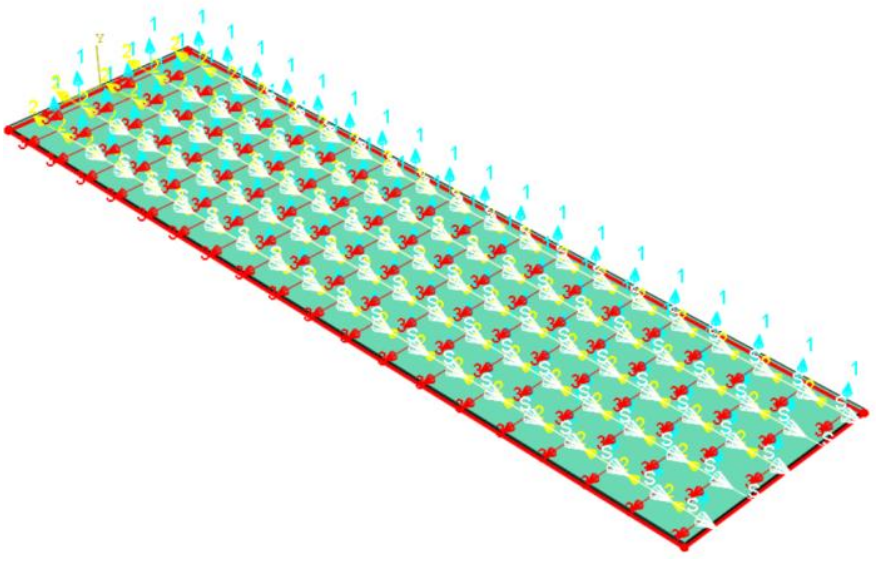

Figura 9: Orientación a $90^{\circ}$ de las fibras en la capa de laminado (Fuente: propia)

\section{Resultados y Discusión de resultados}

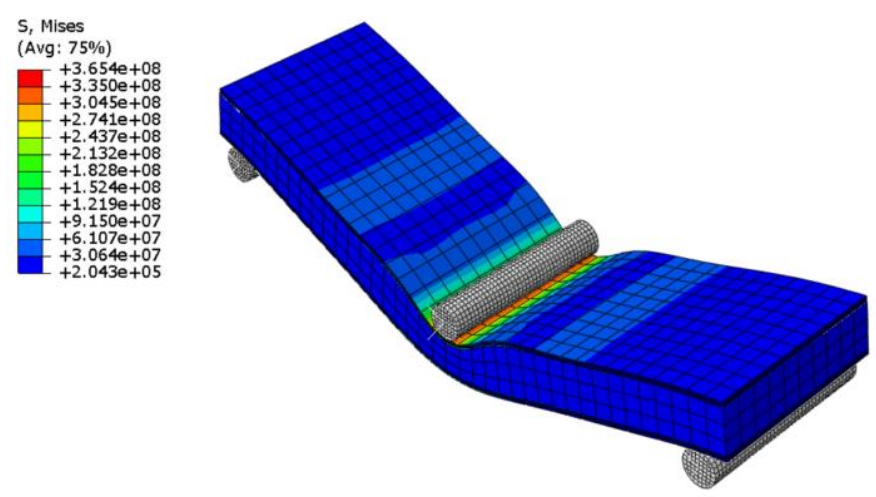

Figura 10: Campo de esfuerzos de Von Mises del compuesto sándwich (Fuente: propia)
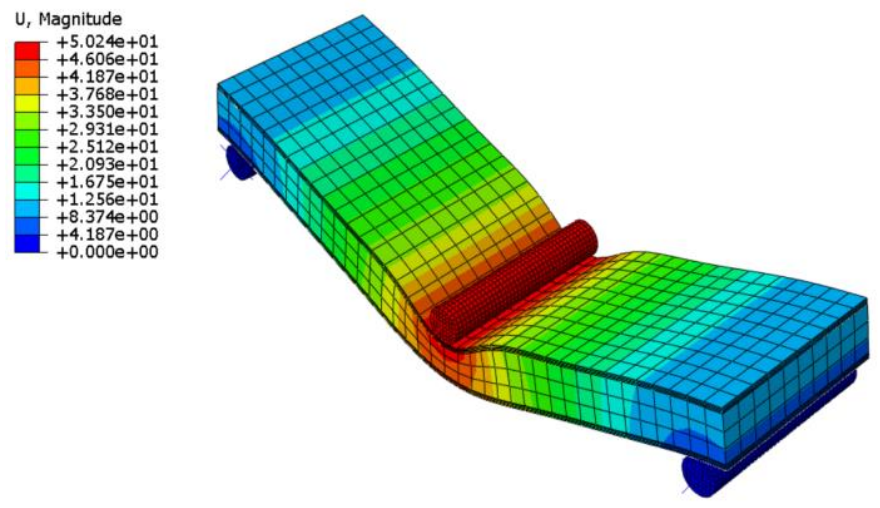

Figura 11: Campo de deformaciones en la estructura sándwich (Fuente: propia) 


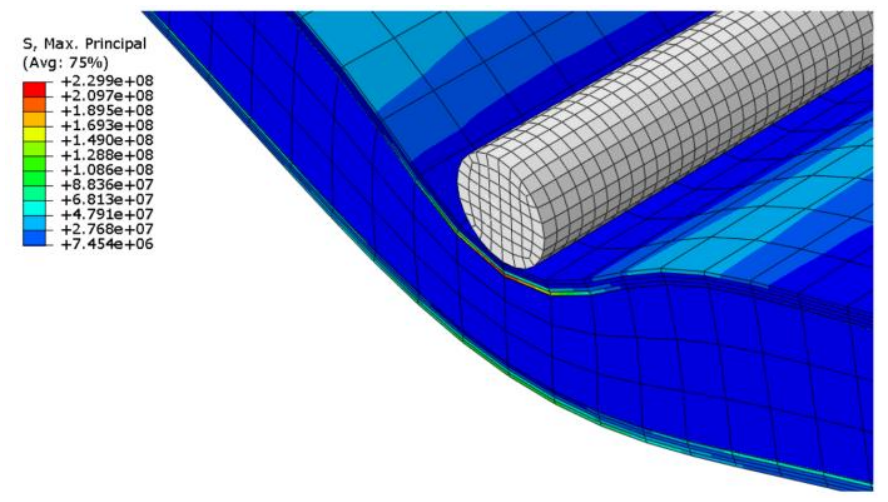

Figura 12: Campos de esfuerzos máximos soportados por las láminas del material sándwich (Fuente: propia)

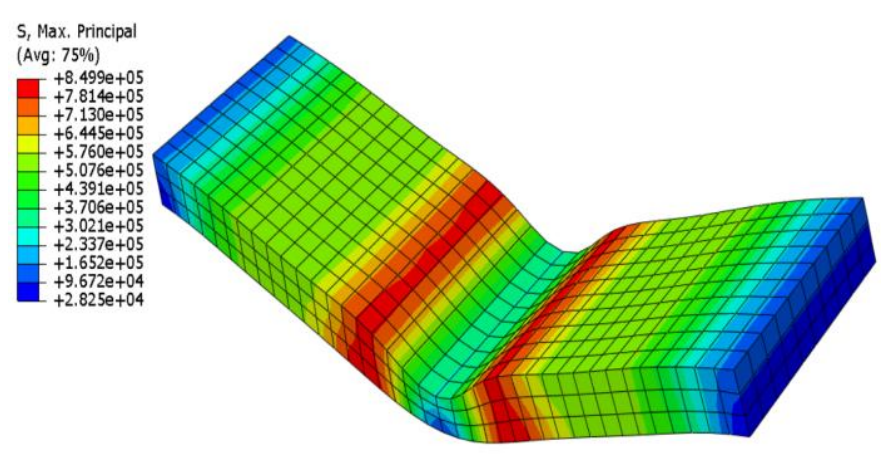

Figura 13: Campos de esfuerzos máximos del núcleo de corcho aglomerado

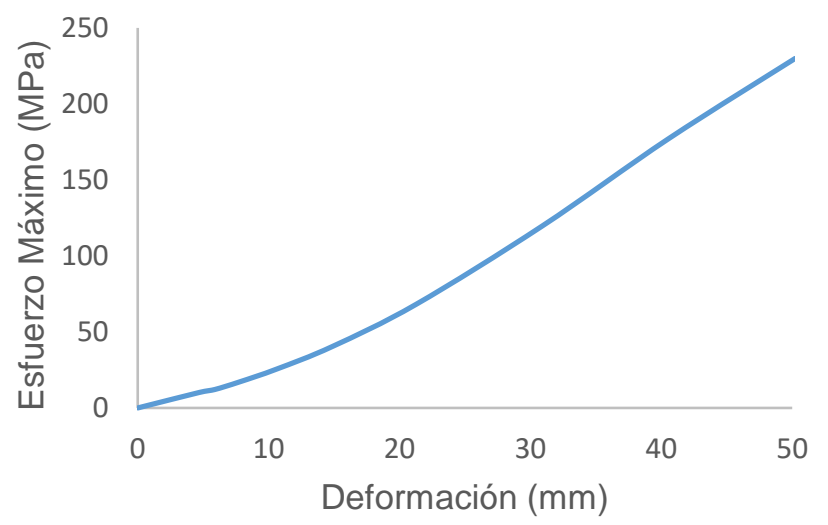

Figura 14: Gráfica esfuerzo máximo-deformación en la cuarta lámina de la piel superior

En esta sección se pueden observar los resultados obtenidos de la simulación computacional del ensayo de flexión. Para una carga con desplazamiento prescrito de $5 \mathrm{~mm}$ se ha comprobado, que la configuración del material puede otorgar muy buenas características estructurales; sin embargo, para una carga con un desplazamiento prescrito de $50 \mathrm{~mm}$ (Figura 11), la configuración del material se llega a fracturar.

Se ha elegido un caso crítico de una carga con desplazamiento prescrito de $50 \mathrm{~mm}$, ya que, en las industrias los materiales suelen estar sometidos a grandes esfuerzos.

Se puede observar en la Figura 12 que los esfuerzos se distribuyen desde la piel superior hacia la piel inferior, debido a que para cada piel hay esfuerzos máximos distintos. Estos esfuerzos máximos tienen una tendencia ascendente (Figura 14), hasta llegar a un pico de $230 \mathrm{MPa}$ aproximadamente.

El núcleo de corcho aglomerado llega a soportar cargas máximas de hasta $850 \mathrm{kPa}$ (Figura 13) para un espesor de $20 \mathrm{~mm}$. Generalmente, el núcleo de un material sándwich tiene baja resistencia; sin embargo, si se realiza un cambio en la configuración y se aumenta el espesor, aprovechando que el corcho aglomerado es un material de baja densidad, es posible alcanzar un compuesto sándwich con alta rigidez a la flexión.

\section{Conclusiones}

Se concluye de los resultados que para la carga a la que ha sido sometida la estructura, conforme se va aplicando la carga se comienza a fracturar, esto se evidencia en el esfuerzo de Von Mises que llega a ser mayor que el esfuerzo máximo en algunas láminas; sin embargo, se observa que, para una deformación de $5 \mathrm{~mm}$, el material cuenta con grandes aptitudes estructurales. Basado en estos resultados, se recomienda que, para próximas investigaciones, se refuerce la estructura incrementando las capas de laminado o los espesores de cada laminado, así mismo con el núcleo, con la finalidad de llegar a obtener un material altamente resistente a los esfuerzos de flexión.

Conforme a lo observado en el campo de esfuerzos máximos, se concluye que el material sándwich natural llega a soportar esfuerzos de $230 \mathrm{MPa}$, lo cual llega a ser equivalente en incluso superior a los esfuerzos máximos que pueden soportar los compuestos sintéticos de fibra de vidrio, que es un valor de 90MPa [8].

Se puede observar en la Figura 12 se distribuyen en todo el material sándwich, lo que implica que las pieles están cumpliendo su función de distribuir las tensiones en todos los laminados; sin embargo, se recomienda realizar análisis de compresión y de corte, ya que, una de las características más importantes de un núcleo sándwich es que debido a que están sometidos a esfuerzos cortantes, este cuente con un adecuado módulo de cizallamiento. 
Finalmente, de los resultados del campo de esfuerzos de Von Mises y del campo de esfuerzos máximos, se puede concluir que las orientaciones de las fibras tienen influencia en el comportamiento estructural de los laminados.

\section{Agradecimientos}

Agradecer a Dios, a mi familia por el apoyo en este hermoso camino de la investigación. Así mismo, agradecer a mi alma máter Universidad Nacional de Ingeniería, por brindarme los conocimientos para poder contribuir al mundo de la investigación y reforzar los valores para ser un excelente profesional.

\section{Referencias}

[1] S.Sivasaravanan and V.K.Bupesh, "Impact properties of epoxy/glass fiber/nano clay composite materials", IOSR-JMCE, 2014, pp 39-41.

[2] A. Lopez-Arraisa et al., "Caracterización y validación mecánica de biocomposites para la construcción de pequeñas embarcaciones. Análisis del ciclo de vida", AEMAC, may. 2017.

[3] S,Mori et al., "Assessment of Ichu Fibers Extraction and Their Use as Reinforcement in Composite Materials", Journal of Natural Fibers, set.2018.

[4] S,Mori et al., "Physical and Thermal Properties of Novel Native Andean Natural Fibers", Journal of Natural Fibers, jul. 2019.

[5] C,Córdova, "Análisis Multi-escala de un material compuesto a base de fibras naturales (Stipa Obtusa) para el diseño de una prótesis transtibial", tesis para obtener el título profesional de ingeniero mecánico, Universidad de Ingeniería y Tecnología, Perú, 2019.
[6] L, Ponce and J, Mantari, "Process of designa an eco-friendly catamarán to extract aquatic plants", Ship Science and Technology, jul. 2019.

[7] O,Castro et al., "Cork Agglomerates as an Ideal Core Material in Lightweight Structures", Material and Design, may. 2009.

[8] J,Paredes et al., "Análisis de las propiedades mecánicas del compuesto de matriz poliéster reforzado con fibra de vidrio 375 y cabuya aplicado a la industria automotriz", UTE, jun. 2017.

[9] E, Monroy et al., "Conceptos básicos de materiales de ingeniería”, 1 era edición, Colombia: Fundación Universitaria Juan de Castellanos, 2019.

[10] G, López, "Correlación entre métodos analíticos y numéricos para el cálculo de vigas y paneles de estructuras tipo Sándwich", tesis para optar al grado de licenciado en ciencias de la ingeniería, Universidad Austral de Chile, Chile, 2002.

[11] S, Brückmann et al., "Functional Integrated Sandwich Structures for Vehicle Concepts of the Next Generation" in International Conference on Material Science and Material Engineering, Chicago, USA, 2014, pp 581-591.

[12] Amorin Cork Composites, Datos Técnicos NL10/NL20/NL25, Portugal, 2013. [En línea]. Disponible en: https://amorimcorkcomposites.com/en/

dsagasteguic@uni.pe 